\title{
BIBECHANA
}

A Multidisciplinary Journal of Science, Technology and Mathematics

ISSN 2091-0762 (online)

Journal homepage: http://nepjol.info/index.php/BIBECHANA

\section{Temperature dependence of the Helmholtz free energy of liquid alkali metals}

\author{
B. K. Singh ${ }^{*}$, Sudhir Singh ${ }^{2}$ \\ ${ }^{1}$ University Department of Physics, T.M. Bhagalpur University, Bhagalpur \\ ${ }^{2}$ Department of Physics, R. D. College Sheikhpura, T.M. Bhag. Univ., Bhagalpur \\ ${ }^{*}$ Corresponding author: E-mail : bijay.k.singh@gmail.com \\ Article history: Received 12 February, 2012; Accepted 5 July, 2012
}

\begin{abstract}
The Gibbs-Bogoliubov variational method has been considered to study the Helmholtz free energy of liquid alkali metals ( $\mathrm{Na}, \mathrm{K}, \mathrm{Rb}$ and $\mathrm{Cs}$ ) as a function of temperature, using Heine Abarenkov type model potential with Hubbard-Sham exchange and correlation function. The computed values are in very good agreement with experimental observations.
\end{abstract}

Keywords: Helmholtz free energy; Entropy; Specific heat; Boltzman constant; Perturbation energy; Madelung energy

\section{Introduction}

The variational prescription based on the Gibbs-Bogoliubov inequality [1] has provided a convenient computational tool for evaluating the free energy of liquid metals with the framework of a hard-sphere reference system and pseudopotential theory [2,3]. Recently, many workers [4-8] have utilized successfully the Gibbs Bagoliubov (GB) variational technique to study the thermodynamic properties of liquid metals and alloys. In most of the cases the earlier studies are confined near the melting temperature (Tm).

The present work is an attempt in this direction to investigate the thermal effect on the Helmholtz free energy of liquid metals for $\mathrm{T}>\mathrm{Tm}$, which is least understood. This will help us to examine the utility of hard-sphere reference system for election-ion interaction energy. We consider the Heine-Abarenkov (HA) form of model potential [9] in conjunction with the Hubbard- Sham [10-11] (HS) exchange and correlation function. For the present purpose we choose $\mathrm{Na}, \mathrm{K}, \mathrm{Rb}$ and Cs because the pseudopotential perturbation can be applied without committing any appreciable error [12].

\section{Theory}

The details of the variational method used for investigating the thermodynamic properties of liquid metals and alloys are available in the work of Umar et al [13] and Ashcroft and stroud [14]. In the framework of GB method the Helmholtz free energy, F, per ion at fixed temperature T and volume $\Omega$ can be expressed as 
B.K. Sing and Sudhir Singh / BIBECHANA 9 (2013) 7-12 : BMHSS, p.8 (Online Publication: Nov., 2012)

$$
\mathrm{F}=\mathrm{F}_{\mathrm{hs}}+\mathrm{F}_{\mathrm{ps}}
$$

Here $\mathrm{F}_{\mathrm{hs}}$ is the Helmholtz free energy of the hard-sphere system, which can be expressed as

$$
\mathrm{F}_{\mathrm{hs}}=(3 / 2) \mathrm{K}_{\mathrm{B}} \mathrm{T}-\mathrm{TS}
$$

where $(3 / 2) \mathrm{K}_{\mathrm{B}} \mathrm{T}$ is the mean kinetic energy and $\mathrm{S}$, the entropy of the system can be written as

$$
\mathrm{S}=\mathrm{Sgas}+\mathrm{S} \eta
$$

with $\quad$ Sgas $=(5 / 2) \mathrm{K}_{\mathrm{B}}+\mathrm{K}_{\mathrm{B}} \ln \left\{\Omega\left(\mathrm{mK}_{\mathrm{B}} \mathrm{T} / 2 \pi \hbar 2\right) 3 / 2\right\}$

$$
\mathrm{S} \eta=\mathrm{K}_{\mathrm{B}} \ln (1-\eta)+3 / 2 \mathrm{~K}_{\mathrm{B}}\{1-(1-\eta) 2\}
$$

Eq. (3) has further been improved by incorporating the low temperature specific heat contribution of electron gas. The resulting expression for the entropy becomes

$$
\mathrm{S}=\mathrm{Sgas}+\mathrm{S} \eta+\mathrm{Selec}
$$

with

$$
S_{\text {elec }}=\pi^{2} K_{B}^{2} T / K_{F}^{2}
$$

where $\mathrm{K}_{\mathrm{B}}$ is the Boltzmann constant and $\mathrm{K}_{\mathrm{F}}$ stand for the Fermi wave vector $\left(K_{F}^{3}=3 \pi^{2} n Z\right.$, $\mathrm{n}$ is the number density).

$$
\mathrm{F}_{\mathrm{ps}}=\mathrm{Feg}+\mathrm{F}_{1}+\mathrm{F}_{2}+\mathrm{Fm}
$$

Where Feg is the free energy of the electron gas, $F_{1}$ and $F_{2}$ are, respectively, first and second order perturbation energies due to the electron-ion interaction. Fm is the Madelung contribution which takes into accounts for the ion-ion interaction. The expressions for these contributions for a metal have been worked out in detail by Harrison [2].

$$
\begin{gathered}
F_{e g}=n z\left[(3 / 10) K_{F}^{2}-(3 / 4 \pi) K_{F}-0.0474-0.0155 \ln K_{F}\right] \\
F_{1}=\lim _{q \rightarrow 0} n z\left\{v(q)-4 \pi z / q^{2}\right\} \\
F_{2}=\left(1 / 16 \pi^{3}\right) \int_{0}^{\infty} v^{2}(q)-s(q)[\{1 / \in *(q)\}-1] q^{4} d q \\
F_{m}=\left(Z^{2} / \pi\right) \int_{0}^{\infty}\{s(q)-1\} d q
\end{gathered}
$$

Where $\mathrm{q}$ is the phonon wave vector $\mathrm{V}(\mathrm{q})$ stands for unscreened from factor, which can be obtained using the HA potential,

$$
V(q)=-\left(4 \pi z n / q^{2}\right) \cos q r_{m}-\left(4 \pi A n / q^{3}\right)\left(\sin q r_{m}-q r_{m} \cos q r_{m}\right)
$$


Here $\mathrm{A}$ is the well depth and $\mathrm{rm}$ is the model radius. These are obtained quantum mechanically by matching the wave function at $r=r_{m} \cdot \in^{*}(q)$ in Eq. (11) is the modified Hartree dielectric screening function which takes into account of the conducting electron interaction,

$$
\epsilon^{*}(\mathrm{q})=1+\{\mathrm{C}(\mathrm{q})-1\}\{1-\mathrm{G}(\mathrm{q})\}
$$

$\in(q)$ is the Hartree dielectric function and $G(q)$ is the correction factor for the exchange and correlated motion of the conducting electron. Presently we consider G(q) prescribed by Hubbard and Sham

$$
G(q)=\frac{1}{2} q^{2} /\left[q^{2}+\left\{2 \pi k_{F}^{3} /\left(0.153+\pi k_{F}\right)\right\}\right]
$$

The structure factor, s(q), for liquid metals appearing in Eq. (11) and (12) can be calculated from the Parcus-Yevick approximation for hard-sphere potential, which is characterized by the hard-sphere diameter $(\sigma)$,or equivalently, by the packing fraction $\eta\left(=\pi \sigma_{M}^{3} / 6\right)$

\section{Results and Discussion}

The general expression for the Helmholtz free energy of pure liquid metals can be written as

$$
\mathrm{F}=\mathrm{Feg}+\mathrm{F}_{1}+\mathrm{F}_{2}+\mathrm{F}_{\mathrm{M}}+(3 / 2) \mathrm{K}_{\mathrm{B}} \mathrm{T}-\mathrm{TS}
$$

The different terms occurring in Eq. (16) are already defined in Section 2. It is well known that hardsphere potential serves as an effective reference system for liquid metals. The best hard sphere reference system is obtained by selecting those diameters which minimize the Helmholtz free energy, $F$, at the same temperature $\mathrm{T}$ and volume $\Omega$ through

$$
\left(\frac{\partial F}{\partial \sigma}\right)_{\Omega, T}=0
$$

The optimum values of the parameters used in the calculation of the Helmholtz free energy of liquid alkali metals as a function of temperature are compiled in Table 1. The volume of the liquid metals at different temperatures required in the calculation is determined from the relation provided in the work of Huijben [17]. The experimental values of the entropies at appropriate temperature are obtained from tables found in Hultgren et al [18-21].

Using Table 1, we can calculate easily the various terms of Eq. (16). The computed values of Feg, $F_{1}, F_{2}$, Fm and Fhs are listed in Table 2. A perusal of Table 2 show that the major contribution to the theoretical determined Helmholtz free energies comes from the Madelung terms Fm, the other terms contribute a relatively small amount and in order of magnitude falls as $F_{1}, F e g, F_{h s}$ and $F_{2}$. Table 2 shows that the magnitude of $F_{1}$ and $F m$ decreases with the rise of temperature where as the magnitude of Feg, $F_{2}, F_{h s}$ increases. The computed values of $\mathrm{F}$ for liquid alkali metals are displayed in Fig.1 as a function of temperature along with the experimental observation of Hultgren et al [18]. The best agreement is obtained for Cs followed by $\mathrm{Rb}, \mathrm{K}$ and $\mathrm{Na}$. It may be noted that the Helmholtz free energy of liquid alkali metals depend sensitively on temperature above the melting point. The rise of temperature 
experiences a decrease in the Helmholtz free energy. Our results reveal that the decrease of magnitude of the Helmholtz free energy due to first order energy and Madelung energy is compensated due to increasing values of $F_{2}$, Feg and $F_{h s}$ as a result $F$ only depends considerably on temperature above the melting point.

Table 1: Input Parameters in the Calculation of the Helmholtz free energy of $\mathrm{Na}, \mathrm{K}, \mathrm{Rb}$ and Cs at different temperatures

\begin{tabular}{|c|c|c|c|c|}
\hline Liquid Metals & $\mathbf{T}\left({ }^{\circ} \mathbf{K}\right)$ & $\Omega($ a.u) & $\sigma($ a.u $)$ & $\mathbf{S} / \mathbf{K B}$ \\
\hline \multirow{5}{*}{$\mathrm{Na}$} & 371.0 & 278.187 & 6.2579 & 7.1043 \\
\hline & 473.0 & 285.722 & 6.1659 & 8.0953 \\
\hline & 573.0 & 293.427 & 6.0922 & 8.8416 \\
\hline & 673.0 & 301.495 & 6.0293 & 9.4533 \\
\hline & 773.0 & 310.077 & 5.9741 & 9.9751 \\
\hline \multirow{5}{*}{$\mathrm{K}$} & 336.6 & 530.504 & 7.6633 & 8.7493 \\
\hline & 423.0 & 543.360 & 7.5449 & 9.6486 \\
\hline & 523.0 & 559.190 & 7.4313 & 10.4569 \\
\hline & 623.0 & 576.037 & 7.3349 & 11.1095 \\
\hline & 723.0 & 593.824 & 7.2502 & 11.6587 \\
\hline \multirow{5}{*}{$\mathrm{Rb}$} & 312.6 & 667.200 & 7.2179 & 10.1972 \\
\hline & 373.0 & 681.292 & 8.1174 & 10.9056 \\
\hline & 473.0 & 705.516 & 7.9788 & 11.8184 \\
\hline & 573.0 & 731.796 & 7.8625 & 12.5406 \\
\hline & 673.0 & 759.994 & 7.7624 & 13.1400 \\
\hline \multirow{5}{*}{ Cs } & 301.8 & 804.117 & 8.7922 & 10.8790 \\
\hline & 373.0 & 824.402 & 3.6678 & 11.7315 \\
\hline & 473.0 & 852.587 & 8.5192 & 12.6487 \\
\hline & 573.0 & 884.556 & 8.3973 & 13.3825 \\
\hline & 673.0 & 919.709 & 8.2915 & 13.9931 \\
\hline
\end{tabular}

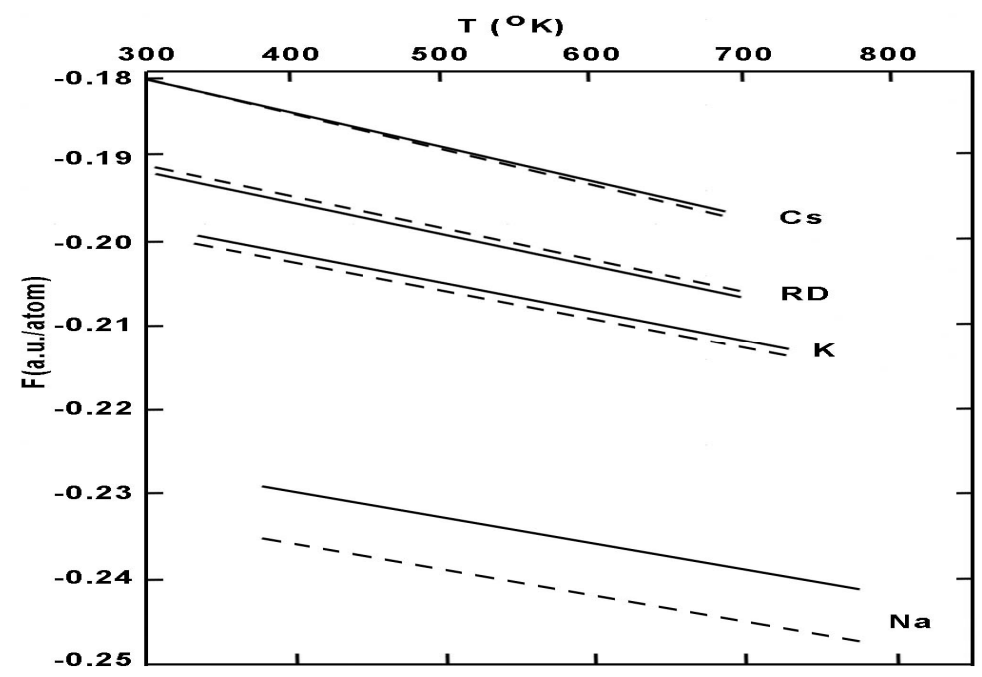

Fig. 1 : Helmholtz free energy of $\mathrm{Na}, \mathrm{K}, \mathrm{Rb}$ and $\mathrm{Cs}$ as a function of temperature. Full and broken curve refer to theoretical and experimental values respectively 
B.K. Singh and Sudhir Singh. / BIBECHANA 9 (2013) 7-12 : BMHSS, p.11 (Online Publication: Nov., 2012)

Table 2: Contribution to Helmholtz free energy of liquid alkali metals as function of temperature

\begin{tabular}{|c|c|c|c|c|c|c|}
\hline $\begin{array}{l}\text { Liquid } \\
\text { Metals }\end{array}$ & $\mathrm{T}\left({ }^{\circ} \mathrm{K}\right)$ & $-F e g$ & $\mathrm{~F}_{1}$ & $-\mathrm{F}_{2}$ & $-\mathrm{Fm}$ & $-\mathrm{F}_{\mathrm{hs}}$ \\
\hline \multirow{5}{*}{$\mathrm{Na}$} & 371.0 & 0.08162 & 0.07734 & 0.00479 & 0.21371 & 0.00656 \\
\hline & 473.0 & 0.08168 & 0.07530 & 0.00619 & 0.20968 & 0.00988 \\
\hline & 573.0 & 0.08175 & 0.07332 & 0.00761 & 0.20575 & 0.01333 \\
\hline & 673.0 & 0.08180 & 0.07136 & 0.00910 & 0.20181 & 0.01695 \\
\hline & 773.0 & 0.08185 & 0.06938 & 0.01066 & 0.19781 & 0.02075 \\
\hline & & & & & & \\
\hline \multirow{5}{*}{ K } & 336.6 & 0.07995 & 0.06419 & 0.00465 & 0.17144 & 0.00773 \\
\hline & 423.0 & 0.07982 & 0.06267 & -0.00587 & 0.16825 & 0.01092 \\
\hline & 523.0 & 0.07966 & 0.06089 & 0.00737 & 0.16454 & 0.01434 \\
\hline & 623.0 & 0.07949 & 0.05912 & 0.00894 & 0.16079 & 0.01896 \\
\hline & 723.0 & 0.07933 & 0.05734 & 0.01057 & 0.15702 & 0.02326 \\
\hline \multirow{5}{*}{$\mathrm{Rb}$} & 312.6 & 0.07826 & 0.05782 & 0.00476 & 0.15837 & 0.00859 \\
\hline & 373.0 & 0.07841 & 0.05779 & 0.00543 & 0.15827 & 0.01111 \\
\hline & 473.0 & 0.07784 & 0.05467 & 0.00747 & 0.15172 & 0.01546 \\
\hline & 573.0 & 0.07757 & 0.05272 & 0.00929 & 0.14753 & 0.02004 \\
\hline & 673.0 & 0.07720 & 0.05076 & 0.01122 & 0.14327 & 0.02481 \\
\hline \multirow{5}{*}{$\mathrm{Ca}$} & 301.8 & 0.07660 & 0.05795 & 0.00419 & 0.14917 & 0.00896 \\
\hline & 373.0 & 0.07638 & 0.05653 & 0.00521 & 0.14641 & 0.01209 \\
\hline & 473.0 & 0.07610 & 0.05466 & 0.00672 & 0.14265 & 0.01670 \\
\hline & 573.0 & 0.07578 & 0.05265 & 0.00835 & 0.13868 & 0.02157 \\
\hline & 673.0 & 0.07545 & 0.05067 & 0.01009 & 0.13466 & 0.02663 \\
\hline
\end{tabular}

\section{Acknowledgement}

We would like to thank Prof. R. N. Singh, Department of Physics, Bhagalpur University for his useful suggestions and encouragement in this work. I am also thankful to the U.G.C., New Delhi, for the financial support under grant no. F10-109/90(RBBII).

\section{References}

[1] A. Isihare, J. Phy A: Gen. Phys, 1 (1968) 53.

[2] W.A. Harrison, Pseudopotential in the theory of metals ,W A Benjamin Inc., New York (1966).

[3] T.E. Feber, Introduction to the theory of liquid metals ,Cambridge: CUP, (1972).

[4] H. Jones, J Chem Phys, 55(1971)2640, Phys Rev A, 133 (1973) 3215.

[5] D. Stroud and N.W. Ashcroft, Phys. Rev. B, 5 (1972) 371.

[6] I.H. Umar and W.H. Young, J Phys F, 4 (1974) 525.

[7] R.N. Singh, J Phys F, 10 (1980) 1411.

[8] R.N. Singh and S. Singh, Physica B, 128 (1985) 304.

[9] V. Heine and I.V. Abarenkov, Philos Mag, 9 (1964) 451.

[10] J. Hubbard, Proc. Roy. Soc. A, 240 (1957) 539.

[11] L.J.Sham, Proc. Roy Soc. A, 283 (1965) 33.

[12] V. Heine and D.L Weare, Solid State Physics, 24 (1970) 249.

[13] I.A. Umar, A. Meyer, M. Watabe and W.H. Young, J. Phys. F, 4(1974) 1691.

[14] N.W. Ashcroft and D. Stroud, Solid State Physics, 33 (1978) 1. 
B.K. Singh and Sudhir Singh / BIBECHANA 9 (2013) 7-12 : BMHSS, p.12 (Online Publication: Nov., 2012)

[15] O. Ese and J.A. Reissland, J Phys F, 3 (1963) 2066.

[16] N.W. Ashcroft and J. Lekner, Phys Rev, 145 (1966) 83.

[17] M.J. Huijben Ph.D. Thesis, University of Groningen, The Netherlands (1978).

[18] R. Hultgren,P.D. Desai, D.T. Hawkin and M. Gleiser, Kelley K K \& Wagmann D D, Selected values of the thermodynamic properties of the elements ,American Society of metals, Metal Park, Ohio, (1973).

[19] D. Adhikari, B.P. Singh, I.S. Jha, and B.K. Singh, J. Non-cryst. Solids, 357 (2011) 2892.

[20] D. Adhikari, I.S.Jha, and B.P.Singh, Philos. Mag. 90 (2010) 2687.

[21] D. Adhikari, B.P. Singh, I.S. Jha, and B.K. Singh , J. Mol. Liq. 156 (2010) 115. 